\title{
Heidegger, le Dasein et l'origine : conversions en vue d'une anthropologie existentiale
}

Albert Piette

\section{OpenEdition}

\section{Journals}

Édition électronique

URL : http://journals.openedition.org/alter/371

DOI : 10.4000/alter.371

ISSN : 2558-7927

Éditeur :

Association ALTER, Archives Husserl (CNRS-UMR 8547)

\section{Édition imprimée}

Date de publication : 1 novembre 2015

Pagination : 46-63

ISBN : 978-2-9550449-1-9

ISSN : $1249-8947$

\section{Référence électronique}

Albert Piette, "Heidegger, le Dasein et l'origine : conversions en vue d'une anthropologie existentiale », Alter [En ligne], 23 | 2015, mis en ligne le 01 décembre 2017, consulté le 01 mai 2019. URL : http:// journals.openedition.org/alter/371; DOI : 10.4000/alter.371 


\title{
HEIDEGGER, LE DASEIN ET L'ORIGINE : CONVERSIONS EN VUE D'UNE ANTHROPOLOGIE EXISTENTIALE
}

\author{
Albert Piette
}

Comment faire de l'existence un thème anthropologique ? Faisons l'hypothèse que Heidegger peut nous aider dans cet objectif d'une anthropologie empirique et théorique de l'existence. Il ne s'agit pas de paraître heideggérien ou anti-heideggérien. Il ne s'agit pas non plus d'expliciter la pensée de Heidegger, ni de l'accepter mais d'aider à comprendre, avec celle-ci et aussi contre celle-ci, cette possible anthropologie, sa spécificité et son thème de travail, la condition humaine. Heidegger, «convertisseur $»^{1}$ de la tradition anthropologique des sciences sociales qui ont toujours contourné le thème de l'existence : pourquoi pas ? Le but de Heidegger n'est bien sûr pas d'établir les bases d'une telle anthropologie ${ }^{2}$ et il a d'ailleurs indiqué à plusieurs reprises son aversion pour l'anthropologie ${ }^{3}$. Cela suppose donc de «convertir » Heidegger lui-même - c'est la deuxième conversion - ou plus précisément le Dasein, sa caractérisation, que Heidegger se refuse de regarder comme un homme concret, substituant à $l^{\prime}$ " ontique » $l^{\prime}$ « ontologique », à l'empirique l'analytique existentiale et les structures fondamentales.

La caractérisation du "Dasein » en un ensemble d' "existentiaux » constitutifs d'une structure de possibilités interpelle l'acte anthropologique mais à la condition $\mathrm{d}^{\prime}$ " empiriciser» ces traits pour qu'ils deviennent pertinents dans une observation de l'existence concrète

\footnotetext{
${ }^{1}$ Cf. C. Malabou, Le change Heidegger. Du fantastique en philosophie, Paris, Léo Scheer, 2004, p. 9.

${ }^{2}$ M. Heidegger, Etre et temps, Paris, Gallimard, 1986, p. 200. Désormais noté ET dans le cours du texte.

${ }^{3}$ J.-C. Monod, " "L'interdit anthropologique" chez Husserl et Heidegger et sa transgression par Blumenberg ", Revue germanique internationale, $n^{\circ} 10$, en ligne, 2009, p. 221-236.
} 
des hommes et que ces observations rendent possible la découverte de nouveaux existentiaux. Cette conversion du Dasein en homme concret interroge à nouveaux frais sur ses caractéristiques. Alors que Heidegger fait de l'inquiétude une caractéristique anthropologique majeure, je tenterai à partir de données évolutionnaires de montrer que la possibilité d'exister des Homo sapiens se situe dans diverses formes de retrait. Ce sera une troisième conversion.

\section{Anthropologie existentiale et homme concret}

Dans cette première partie, nous expliciterons les enjeux des deux premières «conversions ». Aux définitions traditionnelles de l'étant humain, Heidegger reproche de manquer ce qui le différencie des autres étants. Qu'est-ce qui, selon lui, fait l'unité du Dasein, son " entièreté $»^{4}$ ? Dans ce qui constitue traditionnellement le projet anthropologique, la spécificité humaine renvoie à une dimension sociale et culturelle. «La considération du psychique ou mieux du psycho-organique ne suffit pas ici, même pour décrire le complexus entier. Il y faut la considération du social $»^{5}$, précise Marcel Mauss. Et « inversement», ajoute-t-il, soucieux de proposer une appréhension totale des hommes. Mais Heidegger lui reprocherait sa référence aux catégories traditionnelles (biologique, psychologique, etc.) et de surcroît sous l'imprégnation dominante du "socioculturel», comme l'illustre sa célèbre étude sur « les techniques du corps ». Par ailleurs, l'anthropologie comme science de l'homme ne peut être raisonnablement la synthèse d'autres disciplines, «de ce qui peut être exploré de la nature de l'homme en tant qu'il est un être constitué d'un corps, d'une âme et d'un esprit ». Dans ce cas, "la notion de cette science perd toute précision », mentionne Heidegger ${ }^{6}$. Critique encore proférée aujourd'hui à l'endroit d'une anthropologie qui serait trop " générale ».

Chez Heidegger, il est aussi question d'unité et d'entièreté de l'être-au monde. Mais lorsqu'il évoque des caractéristiques existentiales, il indique qu'elles " ne sont pas des pièces à part qui appartiendraient à un composé, l'une d'elles pouvant y manquer momentanément ; au contraire en s'entrecroisant elles forment un tissu solide et original dont l'ensemble constitue l'entièreté du tout structuré que

\footnotetext{
${ }^{4} \mathrm{C}^{\prime}$ est le terme de la traduction que j'utilise (Gallimard).

${ }^{5}$ M. Mauss, Sociologie et anthropologie, Paris, PUF, 1985, p. 329.

${ }^{6}$ M. Heidegger, Kant et le problème de la métaphysique, Paris, Gallimard, 1981, p. 265-266.
} 
nous recherchons » $(E T, 191)$. Au fond, c'est une vision forte de l'humain que dégage Heidegger pour lequel «seul l'homme a en propre cette manière d'être ${ }^{7}$, c'est-à-dire la temporalité. C'est la mort qui se pose ainsi à l'horizon de l'analyse heideggérienne. Le Dasein, l'être "vers la mort», a une relation spécifique à son exister qu'il questionne et son rapport avec la mort se constitue désormais en caractéristique essentielle.

Et Heidegger de reprocher que les sciences, psychologie, anthropologie, sociologie, méconnaissent ce point inconditionnel : la compréhension que le Dasein a de lui-même comme mortel. La saisie de cette dimension est capitale, elle est pour Heidegger «la condition indispensable de toute recherche sur la mort, que celle-ci soit d'ordre historique et biographique ou d'ordre psychologique et ethnologique » (ET, 247). De tels écrits se réduisent, dit Heidegger, à donner des « renseignements » mais manquent « le trépas lui-même », en tant que ce terme désigne "la manière d'être en laquelle est le Dasein par rapport à sa mort ${ }^{8}$.

En posant l'homme comme existence, appréhendée dans son unité comme temporalité, compréhension et mortalité, avons-nous un nouveau socle pour définir l' « objet » de l'anthropologie ? Elle serait, avec l'appui de Heidegger, la science empirique et théorique des « existants» en tant qu'ils existent. Ce serait l'anthropologie existentiale avec sa spécificité : observer l'existence comme entièreté de l'homme. Elle ne serait pas la science correspondant à une « ontologie régionale » visant des étants en particulier, des animaux ou des plantes, ou des secteurs déterminés comme la vie sociale, mais la science correspondant au leitmotiv heideggérien défini par ce que le philosophe nomme $l^{\prime}$ «analytique existentiale » et $l^{\prime}$ « ontologie fondamentale » : l'homme existe, il continue ainsi vers la mort.

Dans cette perspective, l'anthropologie existentiale ne serait pas une discipline régionale comme les autres, elle serait la science fondamentale, entendons un déploiement empirique et théorique de l'ontologie fondamentale, si l'on veut garder le lexique heideggérien. Alors que la sociologie ou l'anthropologie sociale et culturelle serait l'étude de la dimension socioculturelle de l'étant selon ses diverses théorisations et paradigmes, l'anthropologie existentiale étudierait l'existentialité des étants. Elle produirait ainsi des descriptions capables d'être des passerelles vers d'autres disciplines étudiant des sec-

\footnotetext{
${ }^{7}$ M. Heidegger, Questions III et IV, Paris, Gallimard, 1976, p. 80.

${ }^{8}$ ET, 247. Cf. sur ces questions, F. Dastur, Heidegger et la question anthropologique, Paris/Louvain, Peeters, 2003.
} 
teurs limités des étants, comme la sociologie, la psychologie, les sciences cognitives ou la géographie. Ainsi il s'agit de décrire et penser des singularités ontiques, des étants en train d'exister, c'est-à-dire de continuer et de continuer vers la mort. C'est dans cet espace, entre l'infinité des actes individuels d'exister, qu'une anthropologie existentiale réaliserait sa spécificité, méthodologique et conceptuelle: l'observation d'un homme à la fois, en vue de comparaisons et de théorisations.

Nous venons de passer de l'anthropologie sociale et culturelle à l'anthropologie existentiale. Il faut maintenant convertir le Dasein en homme observable. "L'homme, écrit Claude Romano, celui qui naît et qui meurt, celui qui a un corps et possède une différence sexuelle, ne cesse de hanter le Dasein comme un double encombrant dont ce dernier peine à s'affranchir. L'anthropologie continue de hanter l'ontologie fondamentale, en dépit des dénégations réitérées de son auteur. La lecture réputée fautive, qui voit dans l'analytique du Dasein une variante d'anthropologie philosophique, ne peut être simplement un "contresens" $\gg{ }^{9}$. Pierre Hadot me semble encore plus enthousiaste lorsqu'il indique que Heidegger "décrit excellemment ce $\mathrm{qu}^{\prime}$ on appelle le quotidien ${ }^{10}$.

Ce déplacement du Dasein en existant n'est bien sûr pas anodin, il consiste à empiriciser le premier dans le second, à le singulariser comme unité empirique. Considérons que ce que Heidegger pose comme "deux modes d'être » $(E T, 42)$ - en train de se perdre dans l'impropriété et de se retrouver dans la propriété - devient désormais une séquence possible de basculement entre deux activités ou une modulation réciproque à un instant $t$. C'est ce que fait remarquer, en d'autres termes, Catherine Malabou opposée à toute réduction de la pensée heideggérienne à une philosophie de l'identique, insistant de manière très forte sur les glissements d'un mode à l'autre: "entre l'authentique (le propre) et l'inauthentique (l'impropre): modification du souci en préoccupation, modification de la préoccupation en souci, modification du "on" en pouvoir être propre et du pouvoir être propre en quotidienneté »11.

Qu'est-ce qu'impliquerait une anthropologie existentiale? De fait, en vue d'un travail d'observation, il est tentant de considérer ces existentiaux comme des guides heuristiques. Ce point de vue est en

\footnotetext{
${ }^{9} \mathrm{Cl}$. Romano, Au coeur de la raison, la phénoménologie, Paris, Gallimard, 2010, p. 464.

${ }^{10}$ P. Hadot, La philosophie comme manière de vivre, Paris, Albin Michel, 2001, p. 205.

${ }^{11}$ C. Malabou, Le change Heidegger. Du fantastique en philosophie, op. cit., p. 263.
} 
tout cas d'emblée fécond, faisant surgir des nouveaux thèmes d'observation, selon les instants ou la continuité des situations. Ce que Heidegger écrit sur le Dasein constitue alors une lecture à laquelle les anthropologues pourraient être initiés, avant la confrontation empirique, le déploiement de leurs méthodes d'observation et la description d'étants singuliers. Dans cette perspective, il est efficace de considérer que Heidegger propose des «attitudes typiques » ou des "propriétés typiques » de l'existence, «une sorte de récit épuré de $l^{\prime}$ attitude humaine ${ }^{12}$, à confronter aux réalités.

Une anthropologie de l'existence doit ainsi s'enrichir d'observations-descriptions de l'angoisse, du voilement, de l'oubli ou de la non-conscience, de la crainte, de l'attente, de l'ennui et aussi du ne rien faire avec. Elle invite à des descriptions comparées dans des contextes culturels différents mais aussi à des âges différents, des jeunes enfants aux personnes très âgées proches de la mort. Dans cet objectif, Heidegger attribue au Dasein des caractéristiques qui me paraissent essentielles pour répondre à la question : "qu'est-ce que cela fait d'exister? » À l'être quotidien nécessairement «au-monde », donc en situation, il associe certes le souci ou l'angoisse mais aussi l'indifférence, le laisser-aller et la distancialité. Il présente un homme «d'abord et le plus souvent» (ET, 126) - formule adverbiale qui contient une très nette expression empirique - déchargé et dispensé d'être dans un monde déjà là, sorte d'horizon de compréhension préconstituée (ET, 171). Heidegger ne s'y est pas trompé : "Ce côté indifférencié de la quotidienneté du Dasein, écrit-il, n'est pas rien. C'est au contraire un caractère phénoménal positif de cet étant. Toute façon d'exister, telle qu'elle est, provient de ce genre d'être et y revient » $(E T, 43)$. Ces caractéristiques ne sont pas sans connecter à l'homme minimal, que j'ai souvent décrit ${ }^{13}$, qui reporte, ne finit pas, suspend, dont la parcimonie cognitive, la docilité, la fluidité et la distraction viennent mitiger, modérer, modaliser l'enjeu de sens, la préoccupation, le souci, l'exigence et la charge d'existence, l'angoisse, la « résolution » comme manière lucide de « penser jusqu' au bout » $(\mathrm{ET}, 305)$.

Ceci invite à observer, décrire et comprendre les parts mélangées de l'étrangeté, de la tension d'une part, de la familiarité et de la tranquillité d'autre part, telles qu'elles se déploient dans le cours quotidien. Dans quelles situations et à quels moments, quelles attitudes sont pertinentes pour comprendre ce qui se passe? «L'émergence d'un mode n'est possible que par modification d'un autre, sans

\footnotetext{
12 J.-M. Salanskis, Heidegger, Paris, Les Belles Lettres, 1997, p. 17.

${ }^{13}$ Par exemple, A. Piette, Fondements à une anthropologie des hommes, Paris, Hermann, 2011.
} 
déchirure là encore, sans rupture, note Catherine Malabou. Un mode $\mathrm{n}^{\prime \prime}$ "élimine” pas celui dont il prend la place ${ }^{14}$. Les formes de coexistence entre ces différents modes font surgir un grand espace d'observations. L'exercice anthropologique consiste alors à suivre et à décrire un étant humain, à partir de ce qui advient de situation en situation, la succession et aussi la simultanéité de ses modes d'engagement et de relâchement, de lucidité et de voilement, ses modes d'être présent en avant de soi-même, d'être déjà ailleurs par rapport à l'endroit où il est, de ne pas être confronté à soi, les états d'affairement, de préoccupation et de tension, d'activité ou de passivité. Cela implique bien, me semble-t-il, de saisir la différence des états d'esprit, des vécus - qu'il ne faut donc pas négliger malgré la réticence de Heidegger à leur sujet -, proches d'ailleurs des «dispositions » révélant "comment on se sent », "comment on va », avec lesquelles les hommes ressentent et s'éprouvent (ET, 134), entre les "scènes", où se déploient les actions à enjeux et les situations de "coulisses », les transitions entre scènes à enjeux différents. C'est cette pratique d'observation et d'introspection que j'ai nommée "phénoménographie », non sans quelque déplacement avec la position heideggérienne. Le mot a un double intérêt. Par l'étymologie, il désigne l'étude de ce qui apparaît, les formes, les gestes, les mouvements, les postures. Et en lien avec le mot phénoménologie, il désigne une méthode, le contrepoint empirique de la phénoménologie, comme études des vécus, des flux de conscience, des états d'esprit.

Il est d'autres choses que l'homme ne fait pas. L'idée de la mort, il ne l'affronte pas, il l'esquive, il ne la pense pas imminente, il préfère le divertissement et la tranquillité, répète Heidegger. Exister s'accompagne d'une sorte de réserve négative qui prend des modes différents selon les situations. Comment, dans une situation, un être vieillissant ou très malade pense et ne pense pas à la mort ? Comment continuet-il alors à être « vers la mort »? Et celui qui décide et prépare l'acte de se suicider ? Comment est-il ? Comment se mêlent en lui tension et fatigue ? Car il est possible aussi d' « y-voir-clair-en-conscience » (ET, 296) et $d^{\prime}$ " "oser à fond être soi-même » (ET, 298). Quand cela arrive-til ? À l'anthropologue de regarder l'étant en train d'exister en situation, selon ses diverses possibilités, avec les autres, un étant temporel : inconstant dans la dispersion, devançant lucidement la mort, mais aussi assumant un "héritage ", dans la constance de soi, avec sa façon d'être au présent, au passé et au futur, selon la place et

${ }^{14}$ C. Malabou, Le change Heidegger. Du fantastique en philosophie, op. cit., p. 284. 
l'intensité de chacun, pour reprendre quelques-uns des modes explicités par Heidegger ${ }^{15}$.

Il est heureusement noté par Heidegger que «la Daseinanalyse en tant que science ontique serait une nouvelle science $»^{16}$. Comment l'homme jeté au monde, livré au temps, découvrant la temporalité, caractérisé par un ensemble de structures existentiales, existe-t-il concrètement? Telle est en effet la question d'une anthropologie existentiale. Et cet-étant-là, cet étant-ci, il est bien difficile alors de ne pas les voir comme vivant, avec un corps, un sexe, un âge, jeune ou vieux, dans telle ou telle situation de familiarité ou d'épreuve. Si l'analytique existentiale prétend nous éloigner de l'étant naturel, l'anthropologie existentiale opère la démarche inverse. Notre lecture anthropologique de Heidegger est féconde si dans la marge il est sans cesse rappelé qu'il n'existe que des êtres singuliers, des individus réels à regarder tels qu'ils surgissent en situation. C'est dans cet esprit empiriste qu'il importerait $\mathrm{d}^{\prime}$ " anthropologiser » et de « détailler » les thèmes heideggériens.

Comme le montreraient des observations détaillées des divers basculements des situations quotidiennes, ce lien entre ontique et ontologique, l'étant humain le réalise lui-même. Cela en fait même sa caractéristique essentielle: "ce qui distingue ontiquement le Dasein, note Heidegger, c'est qu'il est ontologique » $(E T, 12)$. Il est lui-même un questionneur métaphysique de l'exister. Pierre Hadot note également l'opposition construite par Heidegger « entre le quotidien, le banal, et un état dans lequel on a conscience de l'existence [...], conscience d'être voué à la mort (c'est ce qu'il appelle l'être-pour-lamort), donc conscience de sa finitude ${ }^{17}$. " À ce moment-là, précise Pierre Hadot, l'existence prend un tout autre aspect, qui est d'ailleurs angoissant; peut-être à cause de la mort, mais angoissant aussi à cause de l'énigme que représente le fait d'exister [...]. Je dois préciser que cette opposition entre le quotidien et l'authentique ne signifie absolument pas qu'il faut vivre sans arrêt dans l'authentique. L'homme vit normalement, et, pourrait-on dire, nécessairement dans le quotidien, mais il peut lui arriver quelquefois d'entrevoir l'existence dans une tout autre perspective. Et c'est déjà beaucoup ».

Tel serait l'objet de l'anthropologie existentiale : observer un étant empirique dans ses diverses humeurs ou «tonalités affectives » du

\footnotetext{
${ }^{15}$ F. Dastur, Heidegger et la question du temps, Paris, PUF, 1990, p. 73 et sqq.

${ }^{16}$ M. Heidegger, Séminaires de Zurich, Paris, Gallimard, 2010, p. 285. Cf. sur ce sujet P. Cabestan et F. Dastur, Daseinanalyse, Paris, Vrin, 2011.

${ }^{17}$ P. Hadot, La philosophie comme manière de vivre, op. cit., p. 205.
} 
quotidien, saisir ses propres sauts ontologiques et aussi ses rebasculements dans l'ontique quotidien. L'homme est un étant privilégié, le seul à questionner le fait d'exister, le seul d'où surgit la question de l'être, celui dont l'être fait question, comme George Steiner l'exprime avec netteté : "Ce privilège réside dans le fait qu'il est le seul à éprouver l'existence comme problématique, le seul à être une présence ontique recherchant une relation de compréhension à l'ontologique, à l' "être" lui-même »18. Ce n'est peut-être pas sans raison que j'ai découvert dans le petit livre de Steiner sur Heidegger l'expression: "anthropologie ontologique». Ce point me fait penser aux questions dites métaphysiques des enfants et aux réponses diverses des parents, plus ou moins évasives, les incitant à ne pas nécessairement insister, à « relâcher » leur tension ontologique.

Il me semble ainsi évident qu'une anthropologie existentiale observe en quoi consiste d'être empiriquement un Dasein, ici et là, dans telle situation, à tel moment. L'accent que Heidegger pose sur la dimension temporelle invite nécessairement et prioritairement à un nouveau positionnement méthodologique ne consistant plus à travailler sur des activités et des situations thématiquement choisies mais, soit sur des instants décrits dans les extrêmes détails gestuels et mentaux, soit sur la continuité des activités, des moments et des situations en train de s'enchaîner dans la permanente "préoccupation ». Ceci recadre la focale moins sur les relations et les modes de coordination dans une activité que sur les modalités de présence et aussi les modes de conscience ou de non conscience. Et quand Heidegger associe à cette dimension temporelle du Dasein la capacité $\mathrm{d}^{\prime}$ « être-pas-là » ${ }^{19}$ sur le mode de l'absence, de la dissimulation et de l'oubli, les modes de conscience, de lucidité, les pensées dans l'action, celles qui lui sont associées, autant que les pensées autres, s'imposent comme une thématique empirique importante.

Il serait aussi possible de considérer à partir du discours de Heidegger la dimension relationnelle et sociale du Dasein. Mais à quoi bon lire en sciences humaines et sociales Heidegger si c'est pour revenir à ce point bien accepté et traité couramment? Certes Heidegger présente un Dasein toujours au-delà de lui-même, ouvert à, relationnel, pourrais-je ajouter. "Il n'est d'emblée "trouvable" de luimême, écrit-il, que là où je détourne les yeux des "vécus" et du “centre d'actes" »(ET, 119). Mais du point de vue d'une anthro-

\footnotetext{
${ }^{18}$ G. Steiner, Martin Heidegger, Paris, Flammarion, 2008, p. 109.

${ }^{19}$ M. Heidegger, Les concepts fondamentaux de la métaphysique, Paris, Gallimard, 1992, p. 102 et $s q q$.
} 
pologie existentiale, la bonne interrogation serait pour nous la suivante : quel effet cela fait-il d'être cet étant en relation, avec cette manière d'exister toujours en déplacement, avec quelles tonalités affectives, quels états d'esprit, actions et gestes ?

$\mathrm{Ne}$ sommes-nous pas au cœur de l'anthropologie: la solitude existentiale d'un homme, l'individu, tout en étant au monde, avec les autres? Heidegger ne manque pas de critiquer l'absorption des singularités dans un être-ensemble qu'implique la vie quotidienne sous le monde $d u$ « on » où chacun est l'autre (ET, 126), mais il refuse aussi de penser un moi fermé sur lui-même, valorisant au contraire un étant en rapport, un être-avec. En lien avec des remarques de Françoise Dastur, François Raffoul explicite ce lien entre l'esseulement de l'homme et son ouverture : "Autrui se donne ici sur le fond du caractère unique, non substituable du Dasein. Dans cette mesure, c'est l'être-pour-la-mort - en tant que la mort est ce par quoi le Dasein est à chaque fois mien et seulement à moi puisque personne ne peut mourir à ma place - qui s'avère la possibilité de l'être-avec $»^{20}$.

Pour le dire concrètement, en vue d'une anthropologie existentiale, quand deux individus sont en relation, l'anthropologue s'intéresserait donc à l'un de ceux-ci, à chacun des deux mais séparément, agissant, parlant, interagissant, pensant, sentant, dans ce qui concerne la relation en cours et aussi dans ce qui ne la concerne pas directement. Après la rencontre, les deux individus continuent d'exister, rencontrant chacun d'autres individus. Idéalement, l'anthropologue continue auprès de l'un d'eux. Partir de l'unité empirique n'implique pas nécessairement que celle-ci soit pensée comme sujet ou cogito. Cet individu est en effet toujours en décalage avec lui-même, en avant de lui-même, dirait Heidegger. Ce point est d'ailleurs une difficulté majeure du travail de description des modes d'exister. C'est ce lien entre cet individu si l'on peut dire et ses activités, actions ou paroles qu'il convient de décrire.

Ainsi l'anthropologie existentiale précise son objectif : décrire et penser des solitudes existant et continuant, loin du monde des sciences sociales dans lequel l'individu n'est jamais seul et encore moins singulier, toujours en lien, en connexion, en groupes, en réseaux, dominant les uns, dominé par les autres, appartenant, expertisant, évaluant, etc. Ou encore observer, non pas l'«entre » qui serait la relation ou l'interaction, mais des individus en train d'exister, donc non rivés à une activité, mais dans le temps, c'est-à-dire "suivre le tracé ou la trace d'une existence à la fois totalement déliée et pourtant

${ }^{20}$ F. Raffoul, À chaque fois mien, Paris, Galilée, 2004, p. 198. 
à chaque fois rivée à soi $»^{21}$, ni substance, ni substrat fixe mais une sorte de présence toujours mobile et ouverte.

\section{De l'angoisse néandertalienne à la tranquillité des Sapiens}

Dans cette troisième conversion, une question de Blumenberg s'impose : "comment est-il possible que l'homme ait seulement pu survivre ? ${ }^{22}$ Cela incite à interroger sur ce qui pourrait caractériser l'archéologie préhistorique du Dasein et, en retour, la spécificité des humains. Posons la question autrement: quand les animaux « deviennent des mortels $»^{23}$ ?

Heidegger a écrit des belles pages sur l'ennui, dont il distingue trois formes. La première, sans doute la plus simple, se manifeste quand le temps semble long ${ }^{24}$ : par exemple, lorsqu'il s'agit d'attendre, dans une gare sans attrait un train qui $n^{\prime}$ arrivera que dans quatre heures ${ }^{25}$. $\mathrm{Ou}$ quand notre hominidé attend, dirions-nous en vue d'une préhistoire existentiale, le retour de ses amis partis à la chasse. Il s'ennuie, il n'est pas captivé, il regarde « vers rien de précis » pour se détourner de la lourdeur du temps qui passe trop lentement dans cette situation. Il n'est pas dans une occupation. Il est au contraire, sans enjeu dans cette situation-là, dans $l^{\prime}$ « état d'être laissé vide ${ }^{26}$. Une autre fois, disons plus tard dans le devenir-humain, l'hominidé constate qu'il vient de s'ennuyer à une fête, à un jeu par ailleurs bien réussi, et dans lesquels il s'est amusé. Il cherchait un passe-temps dans la première forme d'ennui. C'est maintenant toute la situation qui est passe-temps. Elle n'avait rien d'ennuyeux. Il s'y retrouve dans une sorte de laisseraller, de «nonchalance » sans que celle-ci le remplisse. Il n'y a pas été comblé. Il a très bien joué son rôle dans la situation mais cela n'a pas empêché un nouveau vide. L'homme, «bien qu'il soit fondamentalement "souci", a aussi cette autre tendance de ne pas être rempli par l'étant ${ }^{27}$. La troisième forme d'ennui, l'ennui profond, est proche de l'angoisse. L'homme y est dans l'indifférence entière aux choses. C'est elle qui constitue le vide: "nous nous trouvons d'une façon ou d'une autre, comme Dasein, entièrement laissés en plan - non pas seulement

\footnotetext{
${ }^{21}$ Ibid., p. 15.

${ }^{22}$ H. Blumemberg, Description de l'homme, Paris, Cerf, 2011, p. 489.

${ }^{23}$ M. Heidegger, Essais et conférences, Paris, Gallimard, 1980, p. 213.

${ }^{24} \mathrm{M}$. Heidegger, Les concepts fondamentaux de la métaphysique, op. cit., p. 125.

${ }^{25}$ Cf. les commentaires d'A. Schnell, De l'existence ouverte au monde fini. Heidegger 1952-1930, Paris, Vrin, 2005, p. 197-226.

${ }^{26}$ M. Heidegger, Les concepts fondamentaux de la métaphysique, op. cit., p. 157.

27 A. Schnell, De l'existence ouverte au monde fini. Heidegger 1952-1930, op. cit. p. 208.
} 
inoccupés par tel ou tel étant, non pas seulement abandonnés par nous-mêmes à tel ou tel point de vue, mais bien délaissés en entier ${ }^{28}$. Désormais incapable de s'occuper, l'homme, écrasé par le temps, n'y cherche plus aucune possibilité. Dans ce cas, l'ennuyeux, c'est « l'êtretemporel comme tel ${ }^{29}$. Et si l'homme ne supportait pas ou plus d'être conscient de lui-même, de se savoir exister et de se rendre compte ainsi, de ressentir qu'il est comme en perpétuel décalage avec luimême, ou, selon les mots de Sartre, qu'il « ne coïncide avec lui-même, dans une adhésion plénière ${ }^{30}$.

Quand donc les hominidés commencent-ils à devenir «mortels » et à comprendre leur finitude ? Considérons que l'indice de ce rapport à la mort est la présence de sépultures. Certes - et Heidegger insiste beaucoup sur ce point - «nous n'éprouvons pas au sens fort de ce verbe le trépas des autres : nous ne faisons tout au plus qu' “y assister" » (ET, 239). Et Heidegger de préciser : "Ce qui est sûr, c'est que semblable expérience ne peut que rester refusée à chaque Dasein en ce qui le concerne lui-même. La mort des autres n'en est que plus impressionnante » (ET, 237). Or les premières sépultures individuelles certaines des Homo sapiens remontent à 120000 ans, celles des Néandertaliens seraient antérieures, peut-être 170000 ans (mais la date pourrait être plus récente), également au Proche-Orient. Celles-ci seraient-elles aujourd'hui les premiers indices d'«être-avec en compagnie du mort» $(\mathrm{ET}, 238)$ ? Il est plus que chronologiquement vraisemblable que des indices antérieurs de rencontres avec la mortalité existent, comme le laisse penser le site espagnol de La Sima de los Huesos avec sa concentration de squelettes d'au moins vingthuit individus datant de plus de $350000 \mathrm{ans}^{31}$.

À Qafzeh (c'est bien une sépulture sapiens, d'il y a environ 90000 ans), la présence d'un morceau de bois d'un cervidé a été interprétée par les préhistoriens comme une offrande funéraire. Il y a aussi un gros bloc de calcaire et des fragments d'ocre rouge, eux en grande quantité, ainsi que, sur la poitrine de l'enfant, des morceaux de coquille d'œufs d'autruche et des traces de feu dont la présence serait fortuite $^{32}$. «Cette sépulture est, peut-être, selon Alban Defleur, la plus

\footnotetext{
${ }^{28}$ M. Heidegger, Les concepts fondamentaux de la métaphysique, op. cit., p. 213.

${ }^{29}$ Ibid., p. 238.

${ }^{30}$ J.-P. Sartre, L'être et le néant, Paris, Gallimard, 1980, p. 112.

${ }^{31}$ Cf. pour une information générale sur ce point, B. Maureille, Les premières sépultures, Paris, Le Pommier, 2004, p. 37-40.

${ }^{32}$ Nous développons le dossier des sépultures du Paléolithique dans L'origine de la croyance, Paris, Berg International, 2013 et aussi, d'une autre manière, dans Avec Heidegger contre Heidegger. Introduction à une anthropologie de l'existence, Lausanne, L'Âge d'homme, 2014.
} 
riche de tout le Paléolithique moyen. La situation du bois de massacre de cerf posé sur les mains, montre qu'il y a eu offrande faite au mort ${ }^{33}$. Trente mille ans plus tôt, près de là, sur le site de Skhul, d'autres sépultures d'Homo sapiens ont été découvertes avec aussi des dépôts d'objets. Ce serait le moment, à Qafzeh ou à Skhul, où un Sapiens a enterré le mort et a déposé près de lui ce qu'il a pu considérer comme une offrande, en se disant à propos du mort: " et s'il avait une nouvelle vie », « le mort (re)vivrait-il ? ». L'élément déclencheur de la constitution du mode d'être humain me semble la capacité de créer des énoncés contradictoires et de penser leur référence possible comme existant. Cela s'appelle la croyance dont je fais l'exclusivité des Sapiens et que les Néandertaliens n'auraient pas pratiquée. Les positions des préhistoriens sont certes loin d'être unanimes.

Ceci est central : $c^{\prime}$ est le commencement de l'histoire du voilement. Nous serions bien au cœur de la manière humaine d'être au monde, de son unité, donc récente : 100000 ans, peut-être moins. C'est l'absence d'offrandes certaines dans les sépultures néandertaliennes qui me pousse dans ce raisonnement, les offrandes pouvant, mais ce n'est pas une certitude, permettre de penser que des hommes offrent aux morts tel objet en vue d'une nouvelle vie. Ce serait croire, c'est-à-dire penser même ponctuellement que c'est vraiment ainsi - que le mort vit ailleurs -, ce serait jeter un assentiment à tel ou tel élément de ce nouveau monde suggéré par ces énoncés, impossible et simulé mentalement. Mais surtout cela suppose une acceptation de ne pas bien comprendre ce qui est sous-entendu, évoqué par le contenu de cette proposition et de l'ensemble du monde auquel elle renvoie, de ne pas trop y réfléchir, de suspendre son sens critique et donc de rester dans une sorte de flou cognitif. Ce qui, sans constituer un ancrage génétique, a pu installer une nouvelle habitude de penser, typiquement humaine, radicalement différente des Néandertaliens. En effet, ceux-ci n'auraient pas créé d'énoncés contradictoires dont ils n'auraient pas été capables avec leur langage limité d'un point de vue syntaxique et articulatoire ${ }^{34}$. Et donc ils n'auraient pas cru à l'existence de choses incroyables, par exemple de morts qui vivent. Il y a incontestablement des avantages évolutionnaires à pratiquer la restriction, la réserve, le «voilement » pourrais-je ajouter avec un mot bien heideggérien. Alors que les Néandertaliens et sans doute les premiers Sapiens ne pratiqueraient pas la

\footnotetext{
${ }^{33}$ A. Defleur, Les sépultures moustériennes, Paris, CNRS, 1993, p.148.

${ }^{34}$ Sur l'hypothèse de la fluidité cognitive, cf. S. Mithen, The Prehistory of the Mind, Londres, Thames and Hudson, 1996 ; S. Mithen, The Singing Neanderthals.The Origins of Music, Language, Mind, and Body, Cambridge, Harvard University Press, 2007, p. 246-265.
} 
détente cognitive car ils n'auraient pas créé les énoncés « incroyables » la stimulant, un avantage sélectif est donné, pourrait-on dire, à ceux qui la pratiquent et l'acceptent. Surgie du rapport de crédulité envers les énoncés religieux, la tolérance au flou cognitif a pu s'étendre dans les autres activités de la vie quotidienne. Elle confirme ainsi dans toutes situations ce mode mineur par lequel l'être humain accepte la présence d'êtres et d'informations extérieures et contradictoires, mais non perturbatrices à l'activité en cours, le déplacement constant d'enjeux de sens, sans requérir une solution, un accord, une clôture, ainsi que l'établissement de parenthèses, parfois très serrées, autour d'une situation ou d'un événement au-delà desquels les comportements et les pensées semblent sans conséquences, comme oubliés. Ce mode de conscience qui voile, qui atténue l'acuité de la présence me semble moins ce qui accompagne la perception immédiate, la représentation $d$ 'images ou l'accomplissement d'actions habituelles sans y penser, que la sous-utilisation de la capacité d'ordre supérieur de la pensée associée à la conscience de soi et de la mort. Et si ce voilement était ce qui a manqué aux Néandertaliens qui eux aussi enterraient leurs morts? Et si les Néandertaliens étaient restés dans l'angoisse de la finitude, dans la difficulté de se relâcher, d'accepter les contradictions, les échecs, les limites du quotidien? Le voilement pratiqué à un certain moment par les Sapiens contrasterait avec le non-voilement du Néandertalien. C'est une nouvelle amortie. Voiler, c'est oublier, c'est ne pas, ne plus y penser. L'hypolucidité est un élément constitutif du mode mineur qui sera d'autant plus possible il y dix ou quinze millénaires que la structuration de la vie sociale est de plus en plus organisée par le marquage matériel des rôles sociaux impliquant leur meilleure stabilité et aussi par la vie sédentaire entourée de champs et de troupeaux. Mais cette minimalité est aussi d'autant plus nécessaire que cette nouvelle sédentarisation, entraînant une vie sociale plus intense, donc potentiellement plus conflictuelle, a besoin de s'équilibrer sur fond de normes et de règles stables (mais arbitraires), de plus en plus sollicitées et rendues de plus en plus visibles par leur inscription sur des supports divers.

Dans cette hypothèse, le langage n'est pas interprété comme réponse au "manque », à la nouveauté ou à la finitude. Je dirais que la rhétorique contradictoire rendue possible par le langage articulé et syntaxique $^{35}$ invite peu à peu à des actes de croyance, à des semiacceptations dont les hommes se satisfont, et qui vont ainsi générer

\footnotetext{
${ }^{35}$ Cf. P. Carruthers, «The cognitive functions of language », Brain and Behavioral Sciences, $\mathrm{n}^{\circ}$ 25, 2002, p. 657-726. Revue en ligne: http://journals.cambridge.org/action/displayAbstract?from Page $=$ online\&aid $=172495 \&$ fulltext Type $=$ RA\&fileId $=$ S0140525X02000122
} 
un mode d'être relâché. Celui-ci, installé dans les autres activités, amortit l'angoisse et permet plus facilement les créations diverses, artistiques, culturelles, techniques. À lire et à relire les travaux des préhistoriens sur les sépultures néandertaliennes, les incertitudes sont grandes et les avis sont contradictoires sur la présence des offrandes ${ }^{36}$. Aucun élément n'est décisif pour trancher. Ceci dit, la découverte d'une offrande n'impliquerait pas nécessairement un croire comme acte mental, comme acte jetant un assentiment. Celui-ci suppose un énoncé contre-intuitif, contradictoire, qui lui-même supposerait une possibilité de mélanger des informations, des espaces par exemple. Selon notre interprétation, c'est la minoration nécessaire à l'acceptation des énoncés religieux qui remplace, en tout cas, permet de modérer la tension, la rigidité et le malaise hautement ressentis par les Néandertaliens et peut-être aussi les premiers Sapiens. Le déclic viendrait de l'infiltration du mode mineur dans la modalité humaine d'être présent.

Notons à ce propos que, dans leurs travaux sur la " psychologie » néandertalienne à partir de l'archéologie cognitive et de recherches en neuropsychologie, Coolidge et Wynn montrent la capacité de l'homme de Néandertal à maîtriser et à répéter des gestes techniques mais insistent aussi sur la difficulté de son attention à éliminer les interférences ${ }^{37}$. Avec une capacité de mémoire de travail plus basse, cette vulnérabilité aux interférences extérieures est plus forte, engendrant des difficultés de se concentrer, d'innover, comme prisonnier des habitudes antérieures, avec une intelligence moins fluide, à la différence des Sapiens qui disposent de ce qui est nommé "enhanced working memory ». Cette différence serait-elle due à un réarrangement neuronal et celui-ci à une mutation génétique entre 50000 et 100000 ans ? Elle donne en tout cas l'avantage d'un meilleur contrôle de l'attention permettant de distinguer les stimuli importants des autres ${ }^{38}$.

Un mode d'être spécifiquement humain aurait donc été généré par la suspension de l'effet de dissonance cognitive que les énoncés religieux impliquent, dont l'homme a perçu le confort et qui va s'étendre à tous les domaines d'activité. Le coût cognitif qui a pu exister dans la tension et l'hésitation face à ces énoncés s'est ainsi transformé, après une mise entre parenthèses de leur effet contra-

\footnotetext{
${ }^{36}$ L'ensemble complet du dossier est repris dans A. Piette, L'origine de la croyance (Paris, Berg International, 2012).

${ }^{37}$ T. Wynn, F. L. Coolidge, "The Expert Neandertal Mind », Journal of Human Evolution, n 46, 2004, p. 477. Revue en ligne : http://www.uccs.edu/Documents/fcoolidg/Neanderthal\%20JHE\% 202004.pdf

${ }^{38}$ Ibid., p. 470.
} 
dictoire, dans une sorte de confort psychologique bénéfique à reproduire aussi dans diverses circonstances de la vie. Ce sont bien les corollaires de la découverte du relâchement cognitif, de la «mise-àpart» qui m'intéressent, plus que les conditions de transmission d'une religion passant aussi par des manifestations d'engagement fort.

L'homme n'a plus été le même et cela est arrivé : un apprentissage à la non-vérification, à la suspension, au report, à une forme de relâchement cognitif qui facilitera aussi l'acceptation de ce que lui est imposé. Une sorte de «capacité négative » selon les termes de John Keats, qui permet aux hommes de rester dans le doute et l'incertitude, sans chercher avec affairement des faits et des raisons. L'acceptation de la demi-connaissance a suivi la recherche des connaissances $^{39}$. Ainsi l'homme a pu survivre, disons continuer. Il aurait pu se rater, il peut encore se rater $^{40}$. C'est arrivé aux Néandertaliens! Leur disparition a eu lieu en Europe il y a 35000 ans alors que les Sapiens n'y étaient pas installés depuis longtemps. Leur cohabitation sur des espaces proches n'y a pas été longue. Avant, elle avait certainement été beaucoup plus longue au Proche-Orient, avec des répartitions d'espaces différents mais aussi des compétitions ${ }^{41}$. L'hypothèse existentiale aurait sa pertinence pour expliquer que l'effet positif du relâchement cognitif ne se réalisait pas encore au ProcheOrient pour les Sapiens - l'acte de croire venant à peine de surgir -, alors qu'en Europe, il était pleinement à l'avantage de ceux-ci.

Heidegger posait trois caractéristiques de cet état déchu des hommes. Le bavardage, celui des palabres et des redites, redisant ce qui a été dit, perdant contact avec le réel (ET, 169), qui ne correspond pas au langage qui informe; la curiosité, plus précisément, le voir pour voir, la préoccupation effrénée, l'intérêt au seul aspect, devenant vite instabilité, dispersion et bougeotte (ET, 173) ; et l'équivoque rendant possible vacillement et indécision. Et du Néandertalien, ses modes de présence, ne sont-ils pas à l'inverse de ses traits? Il est connu pour son langage limité quant à ses capacités syntaxiques et articulatoires, pour une certaine rigidité qui pourrait rendre problématique de combiner et d'enchaîner des activités diverses, et aussi pour sa difficulté, très « rationnelle », faute de fluidité cognitive, d'accepter les incertitudes, les doutes et les « équivoques ». Avec l'homme

\footnotetext{
${ }^{39}$ J. Keats et H. B. Forman, The Letters of John Keats, Whitefish, Kessinger Publishing, 2004, p. 57.

${ }^{40}$ H. Blumenberg, Description des hommes, op. cit., p. 490.

${ }^{41}$ Cf. aussi L. Meignen, "Néandertaliens et hommes modernes au Proche-Orient », in Les Néandertaliens. Biologie et culture, B. Vandermeersch et B. Maureille (éd.), Paris, Éditions du CTHS, 2007, p. 231-234.
} 
de Néandertal qui enterrait ses morts et savait sa finitude, ne serionsnous pas face à un Dasein disons moins déchu, en prise à l'angoisse de la mort, du rien et du néant, que celui des Sapiens avec sa quotidienneté le déchargeant, le dispensant, comme le note Heidegger, d'être (ET, 128) ?

D'après notre hypothèse, les Sapiens sont devenus quotidiens et les Néandertaliens ne l'auraient pas vraiment été, «jetés-dans-la-mort » sans esquives possibles! Ils seraient l'illustration radicale de la « misère » de la condition humaine, et nous sommes l'espèce du quotidien. Heidegger oppose le poète et le penseur, qui mettent à nu la détresse du monde, au "calculateur » qui nivelle, uniformise dans la volatilité $^{42}$. Le Néandertalien serait un penseur, certes laborieux, mais sans doute pas poète car, en plus de la peur de l'effet de vérité de l'art, il n'utiliserait pas encore la rhétorique contradictoire mêlant des registres d'activités et de domaines différents. Et Homo sapiens est loin d'être un simple calculateur. Il a injecté la distance de la distance, l'hypolucidité. Ce qui a pu lui permettre de suspendre l'angoisse du mortel, et qui a déclenché la créativité technique et culturelle tout en maintenant une dose de repos. Entre les Néandertaliens et les Sapiens, ce serait, brièvement dit, la différence évolutionnaire entre le Dasein authentique et le Dasein qui a dévalé. Heidegger considérait ces deux dimensions comme intrinsèques à la structure du Dasein. Il n'y percevait bien sûr pas une évolution, une hominisation. Répétons-le : "La dévalée du Dasein ne doit donc pas non plus être prise comme "déchéance" à partir d'un "état originel" plus pur et plus haut » (ET, 176). En tout cas, l'anthropologie, celle des hommes, est " privative », selon l'expression de Renaud Barbaras ${ }^{43}$. Elle doit l'être à deux niveaux pour comprendre, par rapport aux flux du vivant et à une sorte de "pureté » du savoir animal qui ne sait pas et qui ne se rapporte pas non plus aux choses en tant que telles, l'effet de "puissance contraire » qu'implique la conscience aboutissant progressivement à la conscience de la temporalité ; mais aussi, par rapport à cette nouvelle lucidité de l'être vers la mort, l'effet de restriction que les Néandertaliens n'auraient pas pu acquérir. Une première négation par la conscience de soi générant la conscience de la mort, une seconde négation par l'effet du voilement qui permet relâchement et création mais aussi agitation et passivité.

\footnotetext{
${ }^{42}$ M. Haar, Heidegger et l'essence de l'homme, Grenoble, Jérôme Millon, 1993, p. 230 sqq.

${ }^{43}$ R. Barbaras, Introduction à une phénoménologie de la vie, Paris, Vrin, 2008, chapitre 3 et en particulier, p. 232 sqq.
} 
Heidegger explique donc que le Dasein est déchargé dans sa quotidienneté. Cette dispense ou décharge d'être $(E T, 128)$ s'exprime par une «tendance au moindre effort » $(\mathrm{ET}, 171)$ et « une tranquillisation pour laquelle tout va pour le mieux» $(\mathrm{ET}, 177)$. Il précise que ces caractéristiques ne génèrent pas l'immobilisme mais plutôt « un affairement effréné » (ET, 177-178). Et dans les structures existentiales, la curiosité, le bavardage et les ambiguïtés définissent le Dasein en train de se perdre lui-même, de se perdre à l'écart de lui-même. De l'analyse de la "personnalité » de l'homme de Néandertal, il ressort une psychologie gérant mal les contradictions, une attention en difficulté face à de nouvelles informations ou à des interférences diverses, une résistance à des changements qui solliciteraient des tâches autres que celles qu'il maîtrise, une difficulté à bien évaluer les coûts, et en même temps une endurance à l'épreuve, et aussi peu de violences entre les hommes ${ }^{44}$. Les Néandertaliens ont certes manifesté une intel-

ligence sociale, des capacités techniques, une compréhension de leur environnement et des adaptations dans des espaces ou des climats différents. Mais ce qui leur ferait défaut, c'est ce que justement Homo sapiens a réussi : mélanger les informations et les actions correspondant à différents domaines d'activités, multiplier les interférences entre ceux-ci. Sans doute ont-ils appris les pensées vagabondes. Et on sait leur intérêt pour les morts. Et si eux n'étaient pas tranquilles, confrontés à l'angoisse d'exister, incapables de curiosités, de préoccupations diverses, de dispersions pertinentes, et de créativités? Voici donc notre Homo sapiens le seul être vivant, tranquille dans les préoccupations multiples, à être ici et ailleurs, ici dans diverses activités. Heidegger faisait du «souci » ce qui permet de comprendre le Dasein dans son entièreté : "Le Dasein est pour lui-même dans son être chaque fois en avance » (ET, 191). Évolutionnairement et phénoménographiquement, les strates du repos sont essentielles pour comprendre la présence des hommes.

Sans doute, les singes sont-ils préoccupés et curieux, comme le montrait déjà les expérimentations de Köhler, sans l'angoisse de la mort mais focalisés, dis-tendus entre les sources d'informations ${ }^{45}$, et les Néandertaliens ne seraient pas trop curieux mais surtout angoissés. Et les Sapiens, tranquillisés, allégeant leurs angoisses, sont désormais capables de curiosités diverses. Ce n'est pas l'angoisse, c'est le

\footnotetext{
${ }^{44}$ Cf. S. Mithen, The Prehistory of the Mind, op. cit.

${ }^{45}$ W. Köhler, L'Intelligence des singes supérieurs, Paris, PUF, 1973.
} 
repos ou le relâchement qui est premier dans ce qui fait la spécificité des Sapiens, dans ce qui structure et unifie leur existentialité. C'est aussi le relâchement et l'hypolucidité qui ont permis toute la créativité des hommes, mais aussi la crédulité et l'agitation tous azimuts. Et la conclusion qui nuance la généalogie heideggérienne: c'est l'angoisse qui précède le relâchement des hommes devenus alors Sapiens, qui précède et désormais imprègne leur dispersion créatrice ou moins créatrice. Comment les Humains modernes ont-ils pu exister? Par l'amortie donc, c'est-à-dire cette part latérale et le mode du relâchement : cela même, et non l'angoisse restée néandertalienne, qui fait $l^{\prime}$ «Humain moderne », qui permet qu'il existe comme il est. Qui permet l'activité, aussi la créativité, et en même temps la passivité et la docilité. Homo sapiens est bel et bien un être-minimal. C'est l'amortie par la minimalité, ajouterais-je, qui caractérise l'unité de son mode d'exister.

Dans cette lecture, contrairement à de nombreux récits d'origine ${ }^{46}$, l'hominisation ne serait pas un processus de compensation de manques originels, selon le célèbre modèle du récit de Protagoras dans un dialogue de Platon, décrivant des animaux bien équipés, en même temps que spécialisés et des humains nus, sans protection. Au contraire, c'est un manque par rapport à un plein, qui vient à deux reprises marquer la transformation hominisante: une première fois la capacité des hominidés de «ne rien faire avec » marque un écart par rapport aux chimpanzés préoccupés, et une deuxième fois la capacité de ne pas y penser et d'être indifférent crée un écart par rapport aux autre hominidés et en particulier aux Néandertaliens pris, sans fluidité cognitive, dans la pensée, la conscience et l'angoisse du temps. Cette opération de conversion de Heidegger consistait bien à penser avec et contre son propre discours.

\footnotetext{
${ }^{46}$ Voir à ce sujet É. Bimbenet, L'Animal que je ne suis plus, Paris, Gallimard, 2011, chapitre premier. Nous remercions Étienne Bimbenet d'avoir attiré notre attention sur cette question du «manque».
} 